\title{
Speech Evoked Auditory Brainstem Response in Stuttering
}

\author{
Ali Akbar Tahaei, ${ }^{1}$ Hassan Ashayeri, ${ }^{2,3}$ Akram Pourbakht, ${ }^{1,3}$ and Mohammad Kamali, ${ }^{2,3}$ \\ ${ }^{1}$ Department of Audiology, School of Rehabilitation Sciences, Iran University of Medical Sciences, Tehran 15459-13187, Iran \\ ${ }^{2}$ Department of Basic Sciences in Rehabilitation, School of Rehabilitation Sciences, Iran University of Medical Sciences, \\ Tehran 15459-13187, Iran \\ ${ }^{3}$ Rehabilitation Research Center, School of Rehabilitation Sciences, Iran University of Medical Sciences, Tehran 15459-13187, Iran
}

Correspondence should be addressed to Hassan Ashayeri; ashayerih.neuroscientist@yahoo.com

Received 3 April 2014; Revised 3 July 2014; Accepted 3 August 2014; Published 19 August 2014

Academic Editor: Marjan Jahanshahi

Copyright ( 2014 Ali Akbar Tahaei et al. This is an open access article distributed under the Creative Commons Attribution License, which permits unrestricted use, distribution, and reproduction in any medium, provided the original work is properly cited.

\begin{abstract}
Auditory processing deficits have been hypothesized as an underlying mechanism for stuttering. Previous studies have demonstrated abnormal responses in subjects with persistent developmental stuttering (PDS) at the higher level of the central auditory system using speech stimuli. Recently, the potential usefulness of speech evoked auditory brainstem responses in central auditory processing disorders has been emphasized. The current study used the speech evoked ABR to investigate the hypothesis that subjects with PDS have specific auditory perceptual dysfunction. Objectives. To determine whether brainstem responses to speech stimuli differ between PDS subjects and normal fluent speakers. Methods. Twenty-five subjects with PDS participated in this study. The speech-ABRs were elicited by the 5 -formant synthesized syllable/da/, with duration of 40 ms. Results. There were significant group differences for the onset and offset transient peaks. Subjects with PDS had longer latencies for the onset and offset peaks relative to the control group. Conclusions. Subjects with PDS showed a deficient neural timing in the early stages of the auditory pathway consistent with temporal processing deficits and their abnormal timing may underlie to their disfluency.
\end{abstract}

\section{Introduction}

Developmental stuttering is a subtype of speech fluency disorders characterized clinically by a disruption in the verbal fluency. Despite a great volume of research and various theories, the underlying mechanisms for stuttering are still not fully understood. Although prior to adolescence and with increasing age, the disorder remediates in the majority of the children; there is a subset of stuttering children who do not recover and their stuttering behaviours persist into adulthood. Regardless of gender and handedness, approximately the prevalence of persistent stuttering in adults has been estimated to be about 1\% [1].

Recent advances in neurological studies have shown abnormal neural activity in the auditory and motor areas as well as subcortical regions such as the basal ganglia in developmental stuttering $[2,3]$. Neuroimaging and magnetoencephalography (MEG) studies have demonstrated that subjects with persistent developmental stuttering (PDS) show functional and structural peculiarities in the central nervous system including unusual activation patterns in the auditory and motor areas as well as gyral and planum temporale anomalies [4-7].

These findings were essentially replicated in several subsequent studies. At least, two imaging studies point to structural brain anomalies in PDS subjects. In the first study, Foundas et al. found that PDS is associated with anomalous planum temporale (PT) asymmetry [2]. The PT seems to play an important role in the representation of speech information at the higher levels of auditory processing and is thought to be important in coordinating incoming auditory feedback with speech output. In another study, using a novel imaging method named diffusion tensor imaging (DTI), Sommer et al. found that the white matter tracts in the left operculum in stutterers were less dense than those in controls [8].

Several electrophysiological studies have shown cortical dysfunctions in stuttering. Recent studies have reported mismatch negativity (MMN) abnormalities in PDS subjects 
that are more prominent in the left hemisphere. MMN, another negative component of ERPs, is believed to index automatic process involved in verbal sensory memory. Using simple pure tone stimuli and speech stimuli, Corbera et al. compared the MMN response between subjects with PDS and fluent speakers [9]. Although they found normal MMN responses for tonal stimuli in both groups, PDS subjects showed abnormal left mastoid MMN amplitude for speech stimuli. This finding is consistent with abnormalities primary auditory cortex of the left temporal-parietal region, a region involved in verbal sensory memory. Cuadrado and WeberFox investigated p600 late language related wave in subjects who stutter [10]. They found smaller p600 amplitudes in stutterers, suggesting atypical syntactic processing. Morgan et al. recorded P300 potentials from the left and right hemisphere in 8 stutters and compared the results with 8 matched controls [11]. They found larger p300 amplitudes in $60 \%$ of stutters (5 out of 8 ) in the left hemisphere. In contrast to stutterers in all normal fluent speakers the amplitude of P300 was larger in the right hemisphere.

Stuttering has also been considered as a timing deficit $[12,13]$. Mistiming in stuttering is not restricted to the speech motor areas. Evidence in favor of timing deficits in the auditory cortex was reported by Beal et al. in a MEG study [14]. They measured auditory evoked magnetic fields in PDS subjects and fluent speakers during passive listening and active speaking tasks. Their results showed that adults with PDS had slower cortical timing (longer auditory M100 latencies) compared to fluent speakers, indicating impaired auditory motor integration. Also, according to the suggestion by Kent, subjects who stutter may be poorer at auditory temporal processing [15]. In addition, the result obtained from studies of central auditory processing in stuttering showed that stuttering adults differed from adults who not stutter in some aspects of auditory temporal information processing [9, 16-18].

Several investigators pointed to the auditory brainstem as a possible origin for a central auditory deficit in stutterers [16, $19,20]$. Electrophysiological tests such as auditory brainstem response (ABR) and frequency following response (FFR) have shown to be highly efficient in identifying brainstem timing deficits. A number of papers have offered convincing evidence that shows that the measurement of speech-ABR is a reliable technique for assessing brainstem timing in clinical populations who are suspected of auditory processing impairments [21]. The speech evoked ABR measurements offer a quantitative evaluation of the auditory pathways at the rostral part of the brainstem and are probably the most reliable of brainstem timing measures at this level. Apart from brainstem timing measures the speech-ABR offers invaluable information about pitch and harmonic encoding.

Three studies using synthetic sentence identificationipsilateral competing message (SSI-ICM) observed that stutterers perform worse than nonstutterers [22-24]. In another study, the binaural masking level difference (BMLD) test at $500 \mathrm{~Hz}$ was administered to 10 adults with developmental stuttering [16]. Adults who stutter displayed smaller MLDs relative to controls. This discrepancy suggests that PDS subjects may have a deficit in binaural interaction processing.
The BMLD is the improvement in the discrimination of a signal in the presence of the noise under dichotic listening conditions when the noise or signal deliver out of phase [25]. The encoding of interaural phase difference (ITD) as reflected by BMLD requires temporal processing in the order of microsecond and depends upon the integrity of ITDsensitive neurons in the brainstem nuclei [26]. Both tests are sensitive to brainstem dysfunction and need the subjects to use temporal information. These findings provide further support for an involvement of the brainstem in stuttering. The current study used the speech-ABR to test the hypothesis that subjects with PDS have specific auditory perceptual dysfunction at the level of brainstem.

\section{Methods and Materials}

2.1. Subjects. In this cross-sectional study, twenty-five individuals with PDS (21 male and 4 female; mean age: $24.48 \pm 3.99$ years old; age range: 16-35 years) were recruited from the IRAN Society of Stuttering and the Speech Therapy Department of Iran University of Medical Sciences. Twenty-five fluent subjects (21 male and 4 female; mean age: $22.44 \pm 2.32$ years old; age range: $16-35$ years) served as the control group (Table 2). All volunteers used Persian as a native language and had normal hearing sensitivity (pure tone thresholds) at octave intervals ranging from 250 to $4000 \mathrm{~Hz}(\leq 20 \mathrm{~dB} \mathrm{HL})$ and were free from otological or neurological problems. Both groups matched for education and sex distribution. All subjects were right-handed as checked by the Persian version of Edinburgh handedness questionnaire [27]. The clinical diagnosis of developmental stuttering and the severity of stuttering were assessed by speech language pathologists. Stutterers suffer from disease for more than ten years and none of them had intensive treatment programs for at least last year. Stuttering severity was measured by Persian version of the Stuttering Severity Instrument-3 (SSI-3) and ranged from mild to severe. All stuttering adults met the clinical criteria of developmental stuttering such as word-initial stuttering, presence of anxiety during stuttering, secondary behaviors, adaptation effect, and situational variations and were differentiated from neurogenic stuttering. Subjects with abnormal response to the click-ABR were excluded from the study. All stutterers and control subjects signed informed consent. The study was approved by the Medical Ethics Committee of Iran University of Medical Sciences.

2.2. Stimulus and Recording Parameters. The ABR data were gathered in a suitable room with a low background noise while subjects were watching a videotape. At the time of testing, subjects were positioned in a comfortable chair and were instructed to be motionless. Prior to the speech evoked ABR assessment, responses to the click-ABR were collected for all subjects. For the click evoked ABR two blocks of 2000 sweeps were delivered at $80 \mathrm{~dB} \mathrm{nHL}$ to the right and left ears via insert ear phones (ER-3) at a rate of 13.3/s with alternating polarity and processed over a $10.66 \mathrm{~ms}$ averaging epoch. The recordings were collected using a band pass filter set to $100-3000 \mathrm{~Hz}$. The speech-ABRs were obtained by the 
Bio-logic Navigator Pro AEP System (Version 7) with vertical electrode array of three surface $\mathrm{Ag}-\mathrm{AgCl}$ electrodes (active: $\mathrm{Cz}$; reference: ipsilateral earlobe; ground: forehead) using a band pass filter set to $100-2000 \mathrm{~Hz}$ and were digitally sampled at $12 \mathrm{KHz}$ (1024 points over $85.33 \mathrm{~ms}$ ). The artifact rejection was set online to $\pm 25 \mu \mathrm{V}$ and all impedance electrodes were maintained under $3 \mathrm{KOhms}$. Two blocks of 3000 sweeps were delivered monaurally at $80 \mathrm{~dB}$ SPL to the right ear via insert ear phones (ER-3, Etymotic Research, Elk Grove Village, IL, USA) at a rate of $10.9 / \mathrm{s}$ with alternating polarity and averaged over an $85.33 \mathrm{~ms}$ time window ( $-15 \mathrm{~ms}$ pre-stimulus). The BioMARK responses were elicited by a 5 formant synthesized syllable/da/, with duration of $40 \mathrm{~ms}$, which was produced using a Klatt synthesizer at the rate of $10 \mathrm{kHz}$ (the default stimulus in the Biological system software). The fundamental frequency $(\mathrm{F} 0)$, the first formant $(\mathrm{F} 1)$, the second formant (F2), and the third formant (F3) shift linearly through the duration of the speech stimulus: the F0 and F1 rise from 105$125 \mathrm{~Hz}$ and $455-720 \mathrm{~Hz}$, respectively, while the F2 decreases from 1700 to $1222 \mathrm{~Hz}$. The F3 reduces from 2550 to $2000 \mathrm{~Hz}$. The last two formants (F4 and F5) remain fixed at $3600 \mathrm{~Hz}$ and $4600 \mathrm{~Hz}$.

2.3. Data Analysis. After recording BioMARK responses, all data were converted to a text file using AEP to ASCII (version 1.6.0) and then were transferred to the Brainstem toolbox (Skoe \& Kraus, 2010) for further analysis. This toolbox is a MATLAB-based package for conducting temporal and spectral analyses. Using the brainstem toolbox we determined timing (peaks: V-A-D-E-F-O), composite (VA inter-peak latency, VA inter-peak amplitude, VA slope) and spectral (F0F1-HF) measures. Due to low detectability of the peak C (present in $80 \%$ of subjects), this wave was excluded from statistical analysis.

To achieve a higher confidence in the timing measures, we objectively detected all peaks via automated peak-picking algorithms in the brainstem toolbox. To obtain more detailed information regarding the frequency encoding in the sustained segment of the response, fast Fourier transform (FFT) was employed and the spectral magnitudes of the FFR were measured over a $11.4-40.6 \mathrm{~ms}$ time window in the three ranges of frequencies (F0: $103-121 \mathrm{~Hz}, \mathrm{~F} 1: 454-719 \mathrm{~Hz}$, and HF: $721-1155 \mathrm{~Hz}$ ). Due to the upper limit of phase locking $(1500 \mathrm{~Hz})$ at the level of the rostral brainstem, F2 and the higher formants, which have higher frequencies, were not measured.

The data from two groups were found to be normally distributed as assessed by a Kolmogorov-Smirnov test. So for statistical analysis parametric tests were used. Independent sample $t$-tests were applied to determine any significant differences in the timing, composite, and spectral measures between stutterers and nonstutterers for each of the test variables. A Pearson product-moment correlation was performed to assess the relationship between severity of stuttering as scores indexed in SSI-3 and brainstem timing measures. Data collected by this research were statistically analyzed via SPSS version 17. For all statistical tests, the significance level was set at $P<0.05$.

\section{Results}

Grand average waveforms for the click-ABR are shown in Figure 2. According to normative data for the click-ABR and in line with normative data in our lab the absolute latencies (I-III-V) and the interpeak latencies (I-III, III-V, and I-V) for all groups were within normal limits. Figure 1 displays grand average responses for the speech evoked ABR in the stutterers and in the control group. As shown in Figure 1, stutterers appear to have longer latency values and shallower VA slope than the control group for the transient portion of the speech-ABR. Statistical analysis revealed significant group differences for the wave $\mathrm{V}(t(48)=3.43, P=0.002)$, wave $\mathrm{A}(t(48)=2.83, P=0.008)$, and wave $\mathrm{O}(t(46)=3.66$, $P=0.001)$. Based on independent samples $t$-tests stutterers had longer latencies for the onset and offset peaks relative to controls. $t$-tests comparisons yielded no significant latency differences for peak $\mathrm{D}(t(43)=1.23, P=0.22)$, peak $\mathrm{E}(t(46)$ $=1.63, P=0.11)$, and peak $\mathrm{F}(t(47)=0.59, P=0.55)$. Table 1 represents the mean and the standard deviation for each measure.

Analysis of composite measures (VA duration, VA interpeak amplitude, and VA slope) revealed significant group differences for the VA slope and the stuttering group had a shallower slope compared to the control group $(t(48)=2.42$, $P=0.02$ ).

For measuring the spectral amplitude of the fundamental frequency and its harmonics (F1 \& HF) Fourier analysis was used. Based on fast Fourier analysis of the speech-ABR $(11.4-40.6 \mathrm{~ms})$ the spectral amplitude of F0 $(80-121 \mathrm{~Hz})-\mathrm{F} 1$ $(454-719 \mathrm{~Hz})$ and HF $(721-1155 \mathrm{~Hz})$ did not differ statistically between the two groups $\{\mathrm{F} 0(t(48)=0.060, P=0.95), \mathrm{F} 1$ $(t(48)=-1.84, P=0.07)$, and $\operatorname{HF}(t(48)=-0.956, P=0.34)\}$.

To assess whether the brainstem timing deficit is related to the severity of stuttering, the latency of each peak was correlated with the score from the SSI by means of the Pearson correlation coefficient. There were significant correlations between stuttering severity and the latency of wave A ( $r=$ $0.45, P=0.02)$ as well as stuttering severity and the latency of wave $\mathrm{O}(r=0.84, P=0.000)$.

\section{Discussion}

To our knowledge this is the first study of speech-ABR in persistent developmental stuttering. In this study we investigated brainstem neural synchrony via the speech evoked ABR in a group of adults with persistent developmental stuttering. ABR components (I-V, I-III, and III-V) were equivalent to normative data for all subjects. The main findings of our investigation in the speech-ABR were significantly increased waves $\mathrm{V}, \mathrm{A}$, and $\mathrm{O}$ latencies, as well as a shallower VA slope in the stuttering group relative to the control group. We also found significant correlations between the latencies of transient measures (wave A and wave $\mathrm{O}$ ) and stuttering severity.

In agreement with Kent's (1983) hypothesis the results presented in our study demonstrate that the brainstem response to transient events is less synchronous in PDS subjects as compared to controls. 


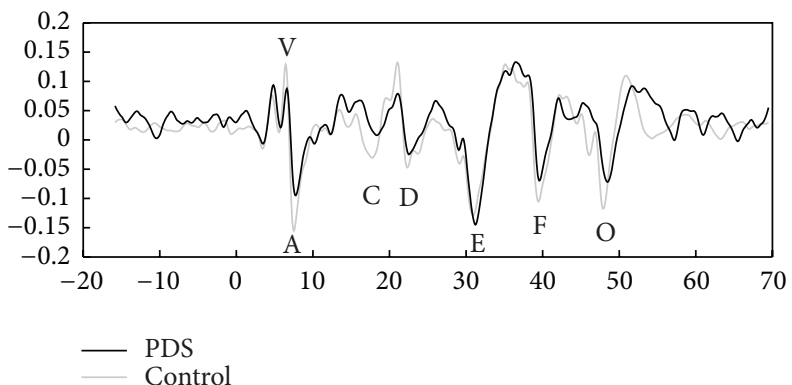

(a)
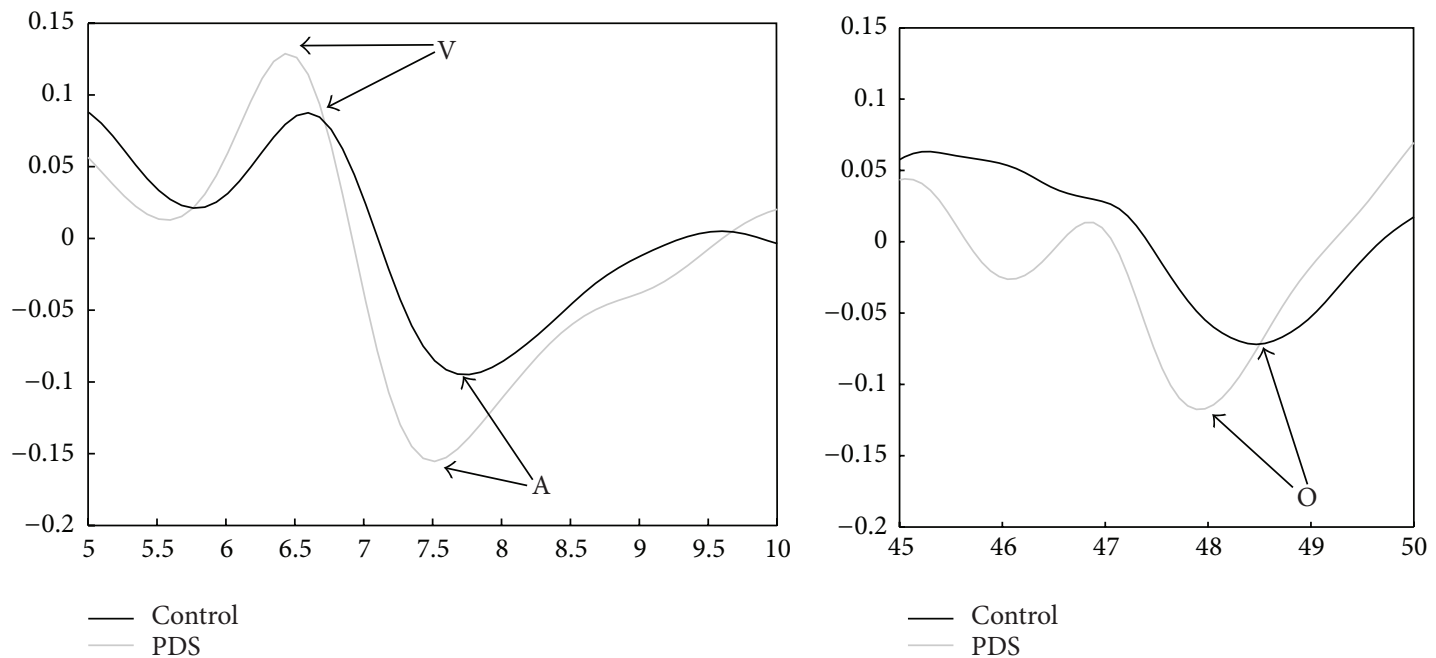

(b)

FIGURE 1: (a) Comparison of brainstem response to speech in controls (gray line) and subjects with persistent developmental stuttering (black line). (b) Auditory brainstem response to speech sounds in stutterers showed significant prolongations in the onset responses (waves $\mathrm{V}$ and A) as well as for the offset response (wave O). PDS = persistent developmental stuttering.

TABLE 1: Mean and standard deviation for latency, composite, and spectral measures.

\begin{tabular}{|c|c|c|c|}
\hline & Control & PDS & $P$ value \\
\hline \multicolumn{4}{|c|}{ Latency measures (ms) } \\
\hline $\mathrm{V}$ & $6.57(0.09)$ & $6.75(0.24)$ & 0.002 \\
\hline A & $7.54(0.18)$ & $7.80(0.41)$ & 0.008 \\
\hline $\mathrm{D}$ & $22.57(0.44)$ & $22.73(0.45)$ & 0.22 \\
\hline $\mathrm{E}$ & $30.91(0.33)$ & $31.09(0.41)$ & 0.11 \\
\hline $\mathrm{F}$ & $39.58(0.46)$ & $39.63(0.44)$ & 0.55 \\
\hline $\mathrm{O}$ & $48.08(0.25)$ & $48.41(0.36)$ & 0.001 \\
\hline \multicolumn{4}{|l|}{ Composite measures } \\
\hline VA duration (ms) & $0.96(0.12)$ & $1.06(0.24)$ & 0.09 \\
\hline $\mathrm{VA}$ amp $(\mu \mathrm{V})$ & $0.31(0.08)$ & $0.28(0.10)$ & 0.21 \\
\hline VA slope (ms/lV) & $-0.33(0.08)$ & $-0.27(0.09)$ & 0.02 \\
\hline \multicolumn{4}{|c|}{ Spectral measures $(\mu \mathrm{V})$} \\
\hline F0 & $0.0480(0.024)$ & $0.0483(0.017)$ & 0.95 \\
\hline $\mathrm{F} 1$ & $0.0088(0.003)$ & $0.0074(0.002)$ & 0.07 \\
\hline $\mathrm{HF}$ & $0.0036(0.001)$ & $0.0032(0.001)$ & 0.34 \\
\hline
\end{tabular}

PDS: persistent developmental stuttering. 


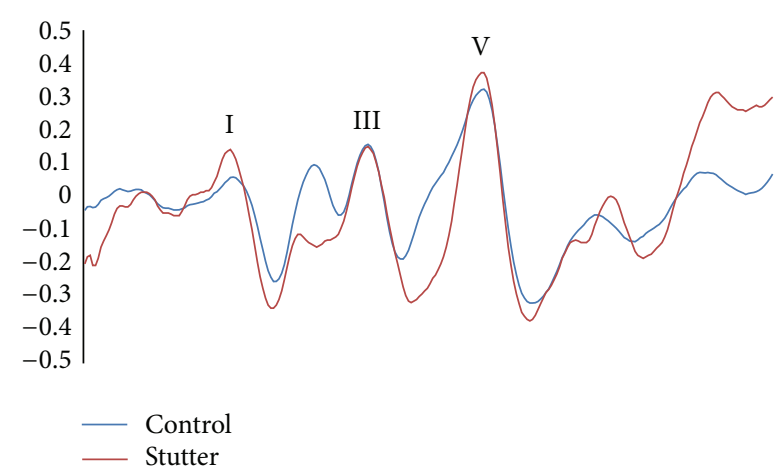

FIGURE 2: Grand average waveforms for the click evoked ABR in stutterers and controls. ABR waves I-V latency interval in stuttering adults was equivalent to normal control subjects.

TABLE 2: Demographic characteristics of stutterers and controls.

\begin{tabular}{lcc}
\hline & Stutterers & Controls \\
\hline Number of subjects & 25 & 25 \\
Gender & $21 \mathrm{M} / 4 \mathrm{~F}$ & $21 \mathrm{M} / 4 \mathrm{~F}$ \\
Age & $24.48 \pm 3.99$ & $22.44 \pm 2.32$ \\
Education & $12.2 \pm 4.2$ & $13.5 \pm 1.2$ \\
\hline
\end{tabular}

4.1. Click Evoked ABR. According to normative data for the click-ABR and in line with normative data in our lab absolute latencies (I-III-V) and interpeak latencies (I-III, III-V, and IV) for all groups (stutterers and control group) were within normal limits. The results regarding theauditory brainstem responses in stuttering are, however, contradictory. Some researchers found significant differences in ABR components between stutterers and nonstutterers $[19,20]$. In contrast and in line with our results, others questioned these findings and failed to show differences in central conduction time of ABRs between stutterers and controls [28, 29]. Some of the disparate findings in this regard are due to differences in the methodology of studies and pathological heterogeneity of stuttering.

Interestingly, this study showed that at least in a subgroup of stutterers, alterations in neural synchrony in the brainstem response to/da/stimuli may occur prior to any significant shifts in the latencies of the click-ABR components. This result indicates that an ABR with normal latency values does not exclude the possibility of timing deficits in the midbrain. Despite the high sensitivity of electrophysiological tests such as ABR in central lesion at the caudal part of the brainstem, they may fail in detecting subtle central auditory deficits at the upper levels. Masuda et al. described a report in which partial inferior colliculus destruction and medial geniculate body degeneration had no effect on the click-ABR [30]. Thus it seems that the speech-ABR gives further information about subcortical information processing complementary to that obtained by the click-ABR [31].

4.2. Speech Evoked ABR. Analysis of timing measures showed statistically significant difference between the two groups for the aperiodic part of the response. The onset peaks (waves A and V) were significantly longer for stutterers versus control group and the majority of subjects in stuttering group had abnormal delay for the offset peak (wave O). The presence of aberrant brainstem timing in PDS subjects demonstrates that the neural response to rapid acoustic transients is less synchronous in PDS subjects as compared to controls.

In our study, adults with PDS showed FFR interwave latency values comparable to that of the control group and comparison of the F0 magnitude between stutterers and nonstutterers yielded no significant magnitude differences. The lack of magnitude differences in the frequency encoding components (fundamental frequency, first formant, and high frequencies: frequencies between F1 and F2) shows that neither pitch nor harmonic encoding is impaired in stuttering.

In line with the results of behavioral investigations of central auditory processing in persistent stuttering, the differences between groups in the onset and offset timing (V-A$\mathrm{O}$ ), but not in the $\mathrm{F} 1$ and HF magnitudes, demonstrate that problem exists in temporal processing rather than spectral encoding. The results of the present study are consistent with Kramer et al. [16]. They found no group differences on the synthetic sentence identification-ipsilateral competing message (SSI-ICM) between stutterers and nonstutterers. The identification of the target sentence in the SSI-ICM largely relies on spectral processing. On the other hand, their findings on the binaural masking level difference demonstrated that adults with persistent developmental stuttering had significantly poorer temporal processing compared to fluent speakers.

Two likely explanations can be described for these temporal abnormalities. One explanation for the shallower slope and longer latencies of the fast onset components of speech-ABR in subjects with PDS may be linked to the timing disturbance in the auditory pathways, which results in asynchronous transmission of auditory afferent information and inefficient processing of stop consonants. Speech encoding requires precise temporal information [9]. Thus, the involvement of the neural generators of onset and/or offset responses within the brainstem might be the cause of brainstem timing deficits in stutterers.

Another explanation is top down influences. Subcortical speech encoding is affected by top down processes (memory, language experience, and attention) through the corticofugal system and such effects can change the response properties of the neurons within the brainstem structures [32]. Further evidence of an association between auditory function at the brainstem and the cortex can be seen in studies showing abnormal brainstem timing in subjects with cortical dysfunction [31]. Since there are projections from the cortex to the rostral and caudal part of the brainstem, it could be inferred that cortical dysfunction leads to aberrant corticofugal feedback on the subcortical regions, which eventually produces impaired neural synchrony at the level of the brainstem. This argument is also supported by animal models, in which ablation of primary auditory cortex changed the neural response properties in the inferior colliculus [32]. Whether asynchronous response to rapid acoustic transients can be related to bottom up mechanisms or result from the top down mechanisms is still debated. It appears reasonable that both 
are engaged, although the range of contribution of each is unknown.

The most likely explanation for different findings of the speech and click evoked responses is that the stimuli used were not comparable. It has been suggested that different mechanisms are likely participated to encode the click versus speech stimuli. Because of backward masking effect (effect of vowel on brief consonant), the speech stimuli may be more challenging to the central auditory system. Discrepancy between the encoding of these stimuli indicates that abnormal timing deficit as revealed by the speech-ABRs is related to differences in synchronization of response generators. The outcomes of the current investigation suggest that PDS subjects have deficient neural timing in response to the onset and offset of speech stimulus. In this study, despite the lack of group differences in the encoding of source cues (information relating F0 or pitch encoding), there were significant differences for some of filter cues (information relating timing and harmonic encoding). These results indicate that stuttering is associated with subtle impairment of speech encoding at the brainstem.

4.3. Clinical Implication. Cognitive factors (such as working memory and attention) are linked to speech perception and production. Considering the effects of high level cognitive processes on the speech-ABR $[33,34]$, we suppose that the speech-ABR can be used as an objective means for monitoring the stuttering remediation following speech fluency shaping programs in subjects who stutter. Further investigation is needed to confirm this assumption. The information gathered from this study can be used to improve our understanding of persistent developmental stuttering and its relationship to auditory abnormality. On the basis of current studies, the brainstem response to the synthesized speech sounds seems to have considerable promise for utilization in clinical populations. Nevertheless, further research is needed before it can receive widespread acceptance.

4.4. Conclusion. These findings demonstrated that adults with persistent developmental stuttering have neural encoding deficits for timing features at the early stages of the auditory pathway. Furthermore, these results provide a positive correlation between stuttering severity and auditory perceptual deficit in developmental stuttering which demonstrate the relevance of speech perception networks to speech production.

\section{Conflict of Interests}

The authors declare that there is no conflict of interests regarding the publication of this paper.

\section{Acknowledgments}

The authors thank Dr. Nahid Jalilvand (Head of Speech Therapy, Department of Iran, University of Medical Sciences) for recruiting patients and Dr. Saeed Farahani and Mr. Hadadzadeh for their cooperation during this study. The authors also thank the students, staff, and patients who took part in this study. The authors are grateful to Dr. Erika Skoe for sending the brainstem toolbox. This study was funded and supported by Iran University of Medical Sciences (IUMS).

\section{References}

[1] E. Yairi and N. Ambrose, "Epidemiology of stuttering: 21st century advances," Journal of Fluency Disorders, vol. 38, no. 2, pp. 66-87, 2013.

[2] A. L. Foundas, A. M. Bollich, D. M. Corey, M. Hurley, and K. M. Heilman, "Anomalous anatomy of speech-language areas in adults with persistent developmental stuttering," Neurology, vol. 57, no. 2, pp. 207-215, 2001.

[3] R. J. Ingham, "Brain imaging studies of developmental stuttering," Journal of Communication Disorders, vol. 34, no. 6, pp. 493-516, 2001.

[4] A. R. Braun, M. Varga, S. Stager et al., "Altered patterns of cerebral activity during speech and language production in developmental stuttering. An $\mathrm{H} 2(15) \mathrm{O}$ positron emission tomography study," Brain, vol. 120, no. 5, pp. 761-784, 1997.

[5] P. T. Fox, R. J. Ingham, J. C. Ingham et al., "A PET study of the neural systems of stuttering," Nature, vol. 382, no. 6587, pp. 158$162,1996$.

[6] S.-E. Chang, M. K. Kenney, T. M. J. Loucks, and C. L. Ludlow, "Brain activation abnormalities during speech and non-speech in stuttering speakers," NeuroImage, vol. 46, no. 1, pp. 201-212, 2009.

[7] A. L. Foundas, A. M. Bollich, J. Feldman et al., "Aberrant auditory processing and atypical planum temporale in developmental stuttering," Neurology, vol. 63, no. 9, pp. 1640-1646, 2004.

[8] M. Sommer, M. A. Koch, W. Paulus, C. Weiller, and C. Büchel, "Disconnection of speech-relevant brain areas in persistent developmental stuttering," The Lancet, vol. 360, no. 9330, pp. 380-383, 2002.

[9] S. Corbera, M. J. Corral, C. Escera, and M. A. Idiazábal, “Abnormal speech sound representation in persistent developmental stuttering," Neurology, vol. 65, no. 8, pp. 1246-1252, 2005.

[10] E. M. Cuadrado and C. M. Weber-Fox, "Atypical syntactic processing in individuals who stutter: evidence from eventrelated brain potentials and behavioral measures," Journal of Speech, Language, and Hearing Research, vol. 46, no. 4, pp. 960976, 2003.

[11] M. D. Morgan, J. L. Cranford, and K. Burk, "P300 event-related potentials in stutterers and nonstutterers," Journal of Speech, Language, and Hearing Research, vol. 40, no. 6, pp. 1334-1340, 1997.

[12] P. A. Alm, "Stuttering and the basal ganglia circuits: a critical review of possible relations," Journal of Communication Disorders, vol. 37, no. 4, pp. 325-369, 2004.

[13] C. L. Ludlow and T. Loucks, "Stuttering: a dynamic motor control disorder," Journal of Fluency Disorders, vol. 28, no. 4, pp. 273-295, 2003.

[14] D. S. Beal, D. O. Cheyne, V. L. Gracco, M. A. Quraan, M. J. Taylor, and L. F. De Nil, "Auditory evoked fields to vocalization during passive listening and active generation in adults who stutter," NeuroImage, vol. 52, no. 4, pp. 1645-1653, 2010.

[15] R. D. Kent, "Stuttering as a temporal programming disorder," in Nature and Treatment of Stuttering: New Directions, R. F. Curlee 
and W. H. Perkins, Eds., College-Hill Press, San Diego, Calif, USA, 1984.

[16] M. B. Kramer, D. Green, and B. Guitar, "A comparison of stutterers and nonstutterers on masking level differences and synthetic sentence identification tasks," Journal of Communication Disorders, vol. 20, no. 5, pp. 379-390, 1987.

[17] A. Hampton and C. Weber-Fox, "Non-linguistic auditory processing in stuttering: evidence from behavior and event-related brain potentials," Journal of Fluency Disorders, vol. 33, no. 4, pp. 253-273, 2008.

[18] P. Howell, S. Davis, and S. M. Williams, "Auditory abilities of speakers who persisted, or recovered, from stuttering," Journal of Fluency Disorders, vol. 31, no. 4, pp. 257-270, 2006.

[19] E. Khedr, W. Abd El-Nasser, E. K. Abdel Haleem, M. S. Bakr, and M. N. Trakhan, "Evoked potentials and electroencephalography in stuttering," Folia Phoniatrica et Logopaedica, vol. 52, no. 4, pp. 178-186, 2000.

[20] I. M. Blood and G. W. Blood, "Relationship between stuttering severity and brainstem-evoked response testing," Perceptual and Motor Skills, vol. 59, no. 3, pp. 935-938, 1984

[21] C. King, C. M. Warrier, E. Hayes, and N. Kraus, "Deficits in auditory brainstem pathway encoding of speech sounds in children with learning problems," Neuroscience Letters, vol. 319, no. 2, pp. 111-115, 2002.

[22] J. W. Hall and J. Jerger, "Central auditory function in stutterers," Journal of Speech and Hearing Research, vol. 21, no. 2, pp. 324337, 1978.

[23] L. F. Molt and A. M. Guilford, "Auditory processing and anxiety in stutterers," Journal of Fluency Disorders, vol. 4, no. 4, pp. 255267, 1979.

[24] M. M. Toscher and R. R. Rupp, "A study of the central auditory processes in stutterers using the synthetic sentence identification (SSI) test battery," Journal of Speech and Hearing Research, vol. 21, no. 4, pp. 779-792, 1978.

[25] G. E. Lynn, J. Gilroy, P. C. Taylor, and R. P. Leiser, "Binaural masking-level differences in neurological disorders," Archives of Otolaryngology, vol. 107, no. 6, pp. 357-362, 1981.

[26] A. Brand, O. Behrend, T. Marquardt, D. McAlpine, and B. Grothe, "Precise inhibition is essential for microsecond interaural time difference coding," Nature, vol. 417, no. 6888, pp. 543$547,2002$.

[27] R. C. Oldfield, "The assessment and analysis of handedness: the Edinburgh inventory," Neuropsychologia, vol. 9, no. 1, pp. 97-113, 1971.

[28] S. V. Stager, "Heterogeneity in stuttering: results from auditory brainstem response testing," Journal of Fluency Disorders, vol. 15, no. 1, pp. 9-19, 1990.

[29] P. W. Newman, K. Bunderson, and R. H. Brey, "Brain stem electrical responses of stutterers and normals by sex, ears, and recovery," Journal of Fluency Disorders, vol. 10, no. 1, pp. 59-67, 1985.

[30] S. Masuda, K. Takeuchi, K. Ukai, H. Tsuruoka, and Y. Sakakura, "Word deafness after resection of a pineal body tumor in the presence of normal wave latencies of the auditory brain stem response," Annals of Otology, Rhinology and Laryngology, vol. 109, no. 12, part 1, pp. 1107-1112, 2000.

[31] J. H. Song, K. Banai, N. M. Russo, and N. Kraus, "On the relationship between speech- and nonspeech-evoked auditory brainstem responses," Audiology and Neurotology, vol. 11, no. 4, pp. 233-241, 2006.
[32] J. Popeláŕ, F. C. Nwabueze-Ogbo, and J. Syka, "Changes in neuronal activity of the inferior colliculus in rat after temporal inactivation of the auditory cortex," Physiological Research, vol. 52, no. 5, pp. 615-628, 2003.

[33] K. Banai, T. Nicol, S. G. Zecker, and N. Kraus, "Brainstem timing: implicationsfor cortical processing and literacy," Journal of Neuroscience, vol. 25, no. 43, pp. 9850-9857, 2005.

[34] B. Wible, T. Nicol, and N. Kraus, "Correlation between brainstem and cortical auditory processes in normal and languageimpaired children," Brain, vol. 128, no. 2, pp. 417-423, 2005. 


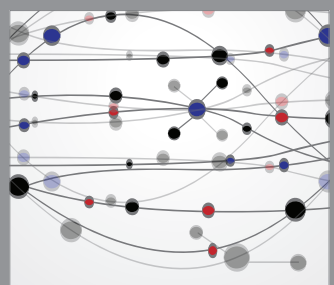

The Scientific World Journal
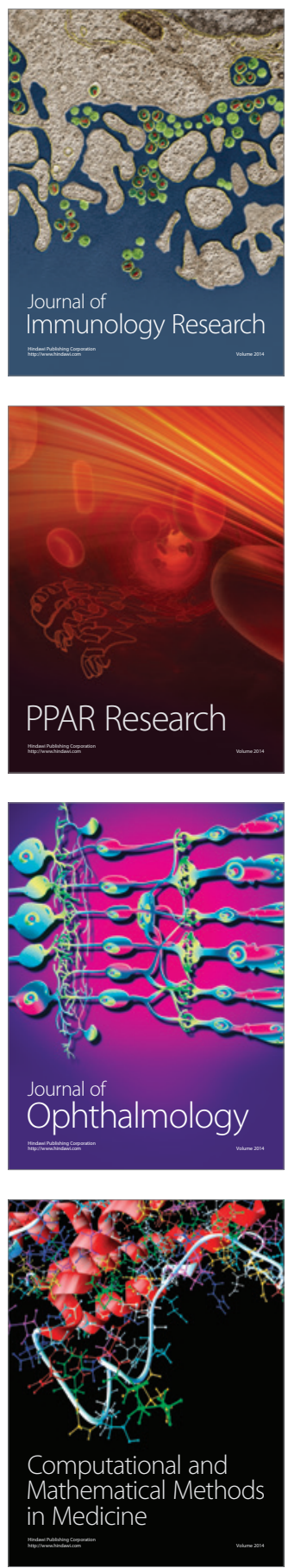

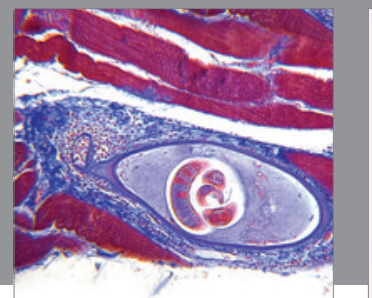

Gastroenterology

Research and Practice
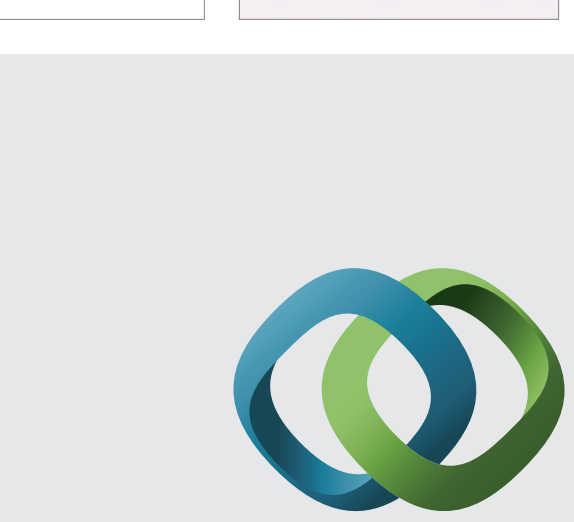

\section{Hindawi}

Submit your manuscripts at

http://www.hindawi.com
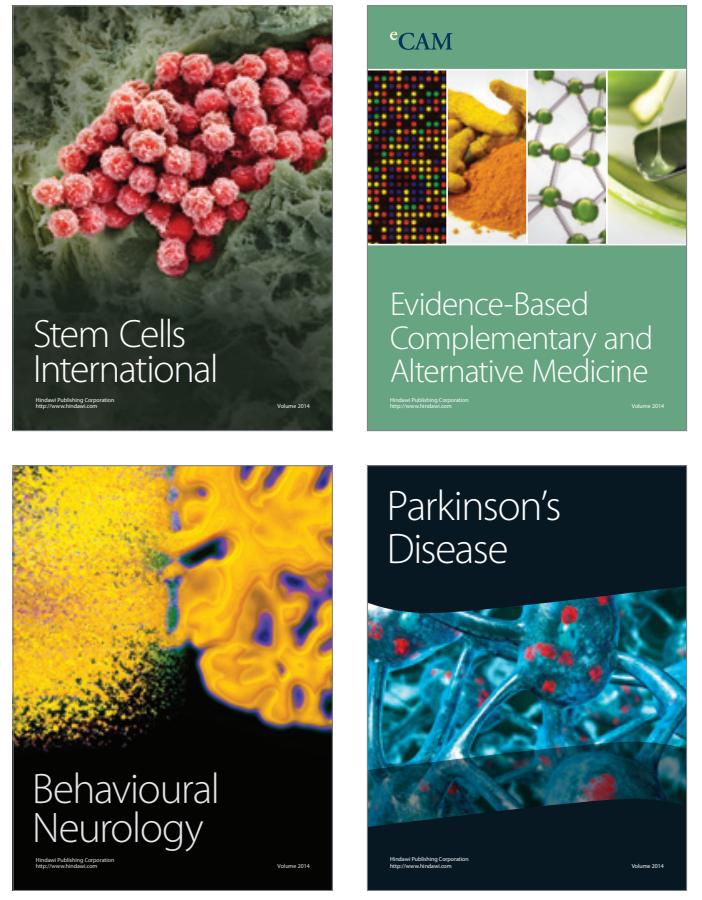
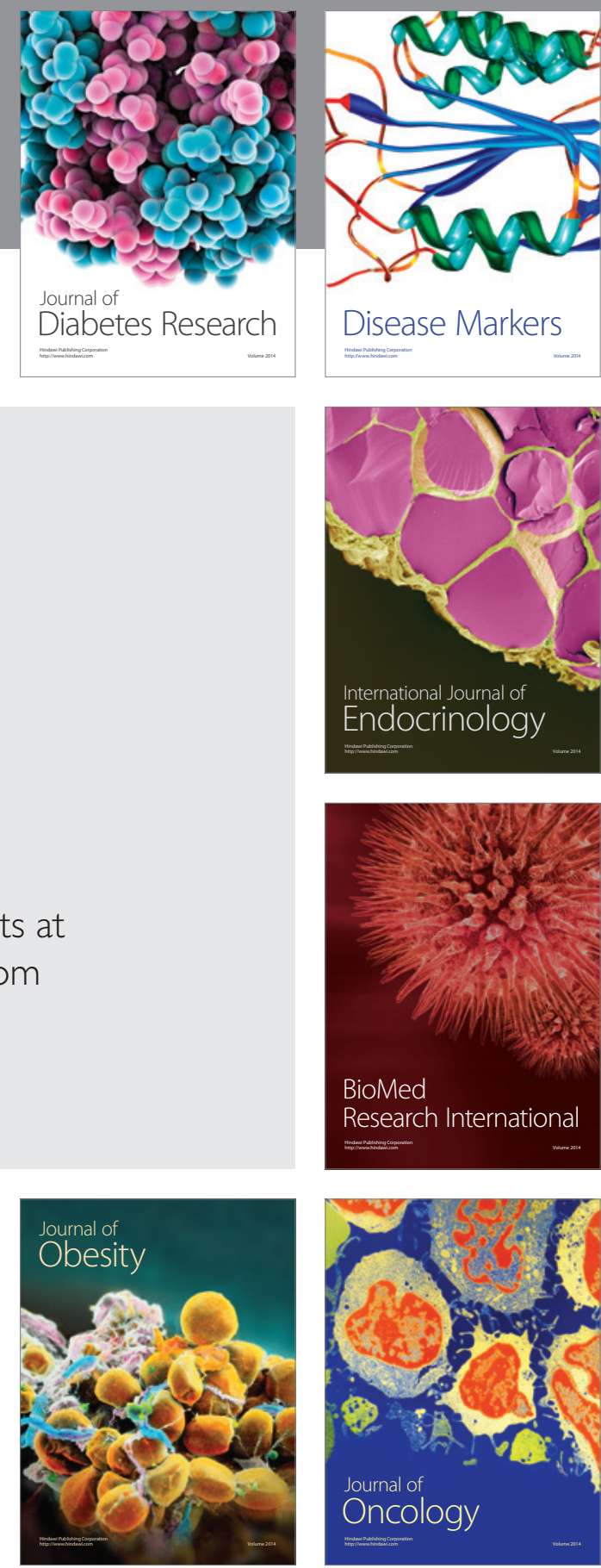

Disease Markers
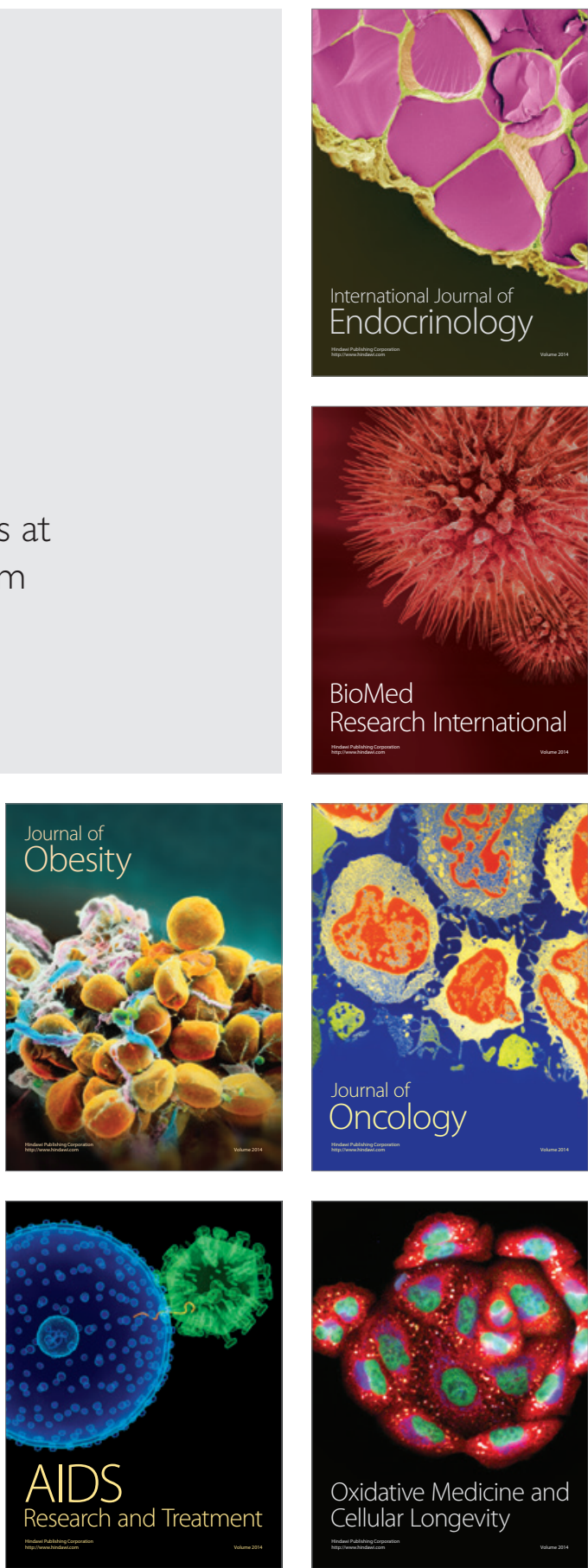